\title{
A STOCHASTIC DIFFERENTIAL REINSURANCE GAME
}

\author{
XUDONG ZENG, ${ }^{*}$ University of Missouri
}

\begin{abstract}
We study a stochastic differential game between two insurance companies who employ reinsurance to reduce the risk of exposure. Under the assumption that the companies have large insurance portfolios compared to any individual claim size, their surplus processes can be approximated by stochastic differential equations. We formulate competition between the two companies as a game with a single payoff function which depends on the surplus processes. One company chooses a dynamic reinsurance strategy in order to maximize this expected payoff, while the other company simultaneously chooses a dynamic reinsurance strategy so as to minimize the same quantity. We describe the Nash equilibrium of this stochastic differential game and solve it explicitly for the case of maximizing/minimizing the exit probability.
\end{abstract}

Keywords: Stochastic differential game; proportional reinsurance; Cramer-Lundberg model; Nash equilibrium; stochastic control

2010 Mathematics Subject Classification: Primary 93E05; 90A09

Secondary 93E20; 60G40; 60J60

\section{Introduction}

For an insurance company, its surplus process can be described by the following classical Cramer-Lundberg model:

$$
R(t)=x+p t-S(t), \quad t \geq 0,
$$

where $p$ is the premium rate, $x$ is the initial reserve, $S(t)=\sum_{i=1}^{N(t)} Z_{i}$ is the total loss, $N(t)$ is the Poisson process of the incoming claims, and $Z_{i}$ is a sequence of independent and identically distributed random variables representing the sizes of the successive claims. Let $S(t)=0$ if $N(t)=0$. When the insurance portfolio is large and an individual claim is relatively small compared to the size of the total reserve, a diffusion which approximates to the CramerLundberg model can be obtained using the following transformation (see [6, Chapter 11]). Assume that $N(t)$ has intensity $m$, and let

$$
\mu=p-m \mathrm{E}[Z], \quad \sigma^{2}=m \mathrm{E}\left[Z^{2}\right] .
$$

In order to find the limit process, assume that $Y:=\alpha Z$ has fixed mean and variance, where $\alpha$ is a scale. Then

$$
m=\frac{\sigma^{2}}{\mathrm{E}\left[Y^{2}\right]} \alpha^{2}, \quad p=\mu+\sigma^{2} \frac{\mathrm{E}[Y]}{\mathrm{E}\left[Y^{2}\right]} \alpha .
$$

Received 20 October 2009; revision received 5 March 2010.

* Postal address: 217 Mathematical Sciences Building, University of Missouri, Columbia, Missouri, 65211, USA.

Email address: zengxu@missouri.edu 
As $\alpha \rightarrow \infty$, one can prove that $R(t)$ converges in distribution to a normally distributed random variable with mean $\mu t$ and variance $\sigma^{2} t$. Therefore, $R(t)$ satisfies the following diffusion model:

$$
\mathrm{d} R(t)=\mu \mathrm{d} t+\sigma \mathrm{d} W(t)
$$

where $W(t)$ is a standard Brownian motion.

In order to reduce the risk of exposure, the insurance company employs a reinsurance strategy. A reinsurer is required to pay a certain fraction of each claim. As a return, the insurance company diverts the same or a larger fraction of all premiums received by the reinsurer. Let $\pi \in[0,1]$ be the fraction of each claim paid by the insurance company. Then $1-\pi$ is the fraction of the claim paid by the reinsurer. Assume that $\lambda(1-\pi)$ is the rate at which the premiums are diverted to the reinsurer. Then we must have $\lambda \geq \mu$. Otherwise the insurance company will make a full reinsurance in order to receive a positive return without any risk. This cannot happen in the real world.

When $\lambda=\mu$, we say that the reinsurance is cheap, that is, the fraction of the premiums diverted to the reinsurer is the same as the fraction of each claim covered by the reinsurer. When $\lambda>\mu$, we say that the reinsurance is noncheap.

The corresponding diffusion approximation for the insurance company with the reinsurance strategy becomes

$$
\mathrm{d} R(t)=(\mu-(1-\pi(t)) \lambda) \mathrm{d} t+\pi(t) \sigma \mathrm{d} W(t)
$$

Suppose that another insurance company also chooses a reinsurance strategy to reduce its risk of exposure. In this paper we consider a stochastic differential game played between the two insurance companies. The game considered here is zero-sum and there is a single payoff function. One company tries to maximize this expected payoff while another company simultaneously tries to minimize the same quantity. The two companies compete by making independent decisions on reinsurance strategies. Their decisions are assumed to be completely observed by both companies.

There are many papers on stochastic differential games in the literature. We follow the line of [2], [3], and [4]. In [2], Browne considered a risky market where the asset prices follow geometric Brownian motions. He studied stochastic differential portfolio games for two investors who have a single payoff function depending on both investors' wealth processes. Earlier, Elliott [3] disclosed that values of stochastic differential games exist if the Isaacs condition holds. For the study of stochastic differential games in insurance and reinsurance, we refer the reader to Suijs et al. [8], who showed that problems in 'non-life' insurance and 'nonlife' reinsurance can be modeled as cooperative games. A nondiffusion model was considered there. This paper, to the author's knowledge, is the first to study stochastic differential games in a diffusion reinsurance model. For this model and relevant optimal strategy selection problems, we refer interested readers to [9], [10], and [12].

The rest of this paper is organized as follows. In Section 2 we introduce a reinsurance model on which a stochastic differential game is defined. In Section 3 we present the FlemingBellman-Isaacs equations for the game with a general payoff function. A verification theorem is also provided in this section. In Section 4 we study a game of maximizing/minimizing the probability for which an explicit solution is obtained. In Section 5, a numerical example is given for the game studied in Section 4. A summary and conclusions are given in Section 6. 


\section{A reinsurance model with competition}

Let $\left(\Omega, \mathcal{F}, \mathcal{F}_{t}, \mathrm{P}\right)$ be a probability space with filtration $\mathcal{F}_{t}$ and two standard Brownian motions $W_{1}(t)$ and $W_{2}(t)$, adapted to $\mathcal{F}_{t}$. The surplus processes of two insurance companies associated with the controls $\pi_{i}:=\left\{\pi_{i}(t)\right\}_{t \geq 0}, i=1,2$, are given by

$$
\mathrm{d} R_{i}^{\pi_{i}}(t)=\left(\mu_{i}-\left(1-\pi_{i}(t)\right) \lambda_{i}\right) \mathrm{d} t+\pi_{i}(t) \sigma_{i} \mathrm{~d} W_{i}(t), \quad i=1,2,
$$

$X_{i}^{\pi_{i}}(0)=x_{i}>0$, where $\lambda_{i} \geq \mu_{i}>0, \sigma_{i}>0, i=1,2$, are constants and $\mathrm{E}\left[W_{1}(t) W_{2}(t)\right]=$ $\rho t$. We call $\left\{\pi_{i}(t)\right\}_{t \geq 0}, i=1,2$, an admissible strategy if $\pi_{i}(t) \in[0,1]$ is $\mathcal{F}_{t}$-adapted. Denote the set of all admissible strategies by $\mathscr{H}$.

In addition, suppose that the insurance companies invest freely in a risk-free asset whose dynamics is governed by the equation

$$
\mathrm{d} B(t)=r B(t) \mathrm{d} t,
$$

where $r$ is the risk-free interest rate. Then the total surplus of insurance company $i$ is the sum of the wealth invested in the risk-free asset and the surplus from the insurance business. Therefore, the total surplus obeys

$$
\mathrm{d} X_{i}^{\pi_{i}}(t)=X_{i}^{\pi_{i}}(t) r \mathrm{~d} t+\left(\mu_{i}-\left(1-\pi_{i}(t)\right) \lambda_{i}\right) \mathrm{d} t+\pi_{i}(t) \sigma_{i} \mathrm{~d} W_{i}(t) .
$$

Without loss of generality, we assume that $x_{1}>x_{2}$, i.e. one company has more initial surplus than the other company. Henceforth, we call the company with the larger initial wealth player 1 , and the other company player 2 . The goal of player 1 is to keep its surplus advantage. Therefore, the objective of player 1 is to maximize a payoff function of the difference $X_{1}^{\pi_{1}}-X_{2}^{\pi_{2}}$ up to some date $\tau$, for example, the date it goes bankrupt (i.e. $X_{1}^{\pi_{1}}(\tau)=0$ ). Simultaneously, player 2 tries to minimize the same payoff.

Let $X^{\pi_{1}, \pi_{2}}(t):=X_{1}^{\pi_{1}}(t)-X_{2}^{\pi_{2}}(t)$. Then $X^{\pi_{1}, \pi_{2}}(t)$ obeys the following stochastic differential equation:

$\mathrm{d} X^{\pi_{1}, \pi_{2}}(t)=\left(D+\pi_{1}(t) \lambda_{1}-\pi_{2}(t) \lambda_{2}+r X^{\pi_{1}, \pi_{2}}(t)\right) \mathrm{d} t+\sigma_{1} \pi_{1}(t) \mathrm{d} W_{1}(t)-\sigma_{2} \pi_{2}(t) \mathrm{d} W_{2}(t)$, where $D=\lambda_{2}-\mu_{2}-\left(\lambda_{1}-\mu_{1}\right)$ and $X^{\pi_{1}, \pi_{2}}(0)=x:=x_{1}-x_{2}>0$.

Denote by

$$
\tau_{y}^{\pi_{1}, \pi_{2}}:=\inf \left\{t>0: X^{\pi_{1}, \pi_{2}}(t)=y\right\}
$$

the first hitting time to the point $y$ under strategies $\pi_{1}:=\left\{\pi_{1}(t)\right\}_{t \geq 0}$ and $\pi_{2}:=\left\{\pi_{2}(t)\right\}_{t \geq 0}$. Let $\tau^{\pi_{1}, \pi_{2}}:=\min \left\{\tau_{l}^{\pi_{1}, \pi_{2}}, \tau_{u}^{\pi_{1}, \pi_{2}}\right\}$ denote the first exit time from the interval $(l, u)$, where $u$ and $l$ are real numbers with $u>l$.

For a given nonnegative function $\eta(z) \geq 0$, a bounded continuous function $c(z)$, and a function $g(z)$, we define the expected payoff function as

$$
\begin{aligned}
J^{\pi_{1}, \pi_{2}}(x)=\mathrm{E}_{x}[ & \int_{0}^{\tau^{\pi_{1}, \pi_{2}}} c\left(X^{\pi_{1}, \pi_{2}}(t)\right) \exp \left(-\int_{0}^{t} \eta\left(X^{\pi_{1}, \pi_{2}}(s)\right) \mathrm{d} s\right) \mathrm{d} t \\
& \left.+g\left(X^{\pi_{1}, \pi_{2}}\left(\tau^{\pi_{1}, \pi_{2}}\right)\right) \exp \left(-\int_{0}^{\tau^{\pi_{1}, \pi_{2}}} \eta\left(X^{\pi_{1}, \pi_{2}}(s)\right) \mathrm{d} s\right)\right] .
\end{aligned}
$$

As usual, we use the convention that $\mathrm{E}_{x}[\cdot]:=\mathrm{E}\left[\cdot \mid X^{\pi_{1}, \pi_{2}}(0)=x\right]$. Then the stochastic differential game consists of player 1 trying to maximize the above payoff function and player 2 trying to minimize it. Let

$$
\underline{J}(x)=\sup _{\pi_{1} \in \mathcal{H}} \inf _{\pi_{2} \in \mathscr{H}} J^{\pi_{1}, \pi_{2}}(x) \text { and } \bar{J}(x)=\inf _{\pi_{2} \in \mathcal{H}} \sup _{\pi_{1} \in \mathscr{H}} J^{\pi_{1}, \pi_{2}}(x)
$$


denote the lower and upper values of the game, respectively. If $\underline{J}(x)=\bar{J}(x)$, the value of the game is given by $G(x):=\underline{J}(x)=\bar{J}(x)$.

\section{Nash equilibrium and Fleming-Bellman-Isaacs equations}

For any admissible $\pi_{2}$ chosen by player 2 , let $\underline{G}\left(x ; \pi_{2}\right)$ be the optimal payoff function of player 1, i.e.

$$
\underline{G}\left(x ; \pi_{2}\right)=\sup _{\pi_{1} \in \mathscr{H}} J^{\pi_{1}, \pi_{2}}(x) .
$$

Assume that $\underline{G}\left(x ; \pi_{2}\right) \in C^{2}((l, u)) \cap C^{1}([l, u])$. Then, with other essential assumptions, $\underline{G}\left(x ; \pi_{2}\right)$ satisfies the following Hamilton-Jacobi-Bellman (HJB) equation (see [5] or [7]):

$$
\sup _{\pi_{1} \in[0,1]} L^{\pi_{1}, \pi_{2}} \underline{G}\left(x ; \pi_{2}\right)+c(x)-\eta(x) \underline{G}\left(x ; \pi_{2}\right)=0, \quad x \in(l, u),
$$

where $L^{\pi_{1}, \pi_{2}}$ is the differential operator, i.e. for any $f(x) \in C^{2}((l, u))$,

$$
L^{\pi_{1}, \pi_{2}} f(x):=\left(D+\pi_{1} \lambda_{1}-\pi_{2} \lambda_{2}+r x\right) f_{x}+\left(\frac{\sigma_{1}^{2}}{2} \pi_{1}^{2}+\frac{\sigma_{2}^{2}}{2} \pi_{2}^{2}-\sigma_{1} \sigma_{2} \rho \pi_{1} \pi_{2}\right) f_{x x} .
$$

Similarly, let $\bar{G}\left(x ; \pi_{1}\right)$ be the optimal payoff function of player 2 with respect to any admissible strategy $\pi_{1}$ chosen by player 1 , i.e.

$$
\bar{G}\left(x ; \pi_{1}\right)=\inf _{\pi_{2} \in \mathscr{H}} J^{\pi_{1}, \pi_{2}}(x) .
$$

Then $\bar{G}\left(x ; \pi_{1}\right)$ satisfies another HJB equation:

$$
\inf _{\pi_{2} \in[0,1]} L^{\pi_{1}, \pi_{2}} \bar{G}\left(x ; \pi_{1}\right)+c(x)-\eta(x) \bar{G}\left(x ; \pi_{1}\right)=0, \quad x \in(l, u) .
$$

A pair of strategies $\left(\pi_{1}^{*}, \pi_{2}^{*}\right)$ is said to achieve a Nash equilibrium or, equivalently, a saddle point for the game if the following inequalities are satisfied. For all $\pi_{1}, \pi_{2} \in \mathcal{H}$,

$$
J^{\pi_{1}, \pi_{2}^{*}}(x) \leq J^{\pi_{1}^{*}, \pi_{2}^{*}}(x) \leq J^{\pi_{1}^{*}, \pi_{2}}(x), \quad x \in[l, u]
$$

If the game has a saddle point, $\left(\pi_{1}^{*}, \pi_{2}^{*}\right)$, then it is easy to check that

$$
\underline{G}\left(x ; \pi_{2}^{*}\right)=\bar{G}\left(x ; \pi_{1}^{*}\right),
$$

and the value of the game $G(x)=J^{\pi_{1}^{*}, \pi_{2}^{*}}(x)=\underline{G}\left(x ; \pi_{2}^{*}\right)=\bar{G}\left(x ; \pi_{1}^{*}\right)$. According to (1) and (2), $G(x)$ satisfies the following (Fleming-Bellman-Isaacs) equations:

$$
\begin{aligned}
& \sup _{\pi_{1} \in[0,1]} L^{\pi_{1}, \pi_{2}^{*}} G(x)+c(x)-\eta(x) G(x)=0, \\
& \inf _{\pi_{2} \in[0,1]} L^{\pi_{1}^{*}, \pi_{2}} G(x)+c(x)-\eta(x) G(x)=0,
\end{aligned}
$$

with the boundary conditions $G(l)=g(l)$ and $G(u)=g(u)$.

The above discussions leading to (4) and (5) are heuristic. We prove the following verification theorem (similar to Theorem 5.1.1 of [11] or Theorem B-1 of [1]) which guarantees that a suitable solution to (4) and (5) is identical to the value function of the game. 
Theorem 1. If there exist $V(x) \in \mathcal{C}^{2}((l, u)) \cap \mathcal{C}([l, u])$ and a pair of real numbers $\pi_{1}^{*}=\pi_{1}^{*}(x)$, $\pi_{2}^{*}=\pi_{2}^{*}(x)$ satisfying

$$
\begin{aligned}
& \pi_{1}^{*}=\arg \sup _{\pi_{1} \in[0,1]} L^{\pi_{1}, \pi_{2}^{*} V(x)+c(x)-\eta(x) V(x),} \\
& \pi_{2}^{*}=\arg \inf _{\pi_{2} \in[0,1]} L^{\pi_{1}^{*}, \pi_{2}} V(x)+c(x)-\eta(x) V(x),
\end{aligned}
$$

such that, for $x \in(l, u), V(x), \pi_{1}^{*}$, and $\pi_{2}^{*}$ satisfy (4) and (5) with the moment property

$$
\int_{0}^{t} \mathrm{E}\left[V_{x}^{2}\left(X^{\pi_{1}, \pi_{2}}(s)\right)\right] \mathrm{d} s<\infty \text { for all } \pi_{1}, \pi_{2} \in \mathcal{H},
$$

then $V(x)$ is the value of the game achieved at the Nash equilibrium strategy $\left(\pi_{1}^{*}, \pi_{2}^{*}\right)$.

Proof. We use the same idea as that given in [2]. Suppose that $V(x)$ satisfies (4), (5), and (7). Write $\Gamma\left(t ; \pi_{1}, \pi_{2}\right)=\exp \left(-\int_{0}^{t} \eta\left(X^{\pi_{1}, \pi_{2}}(s)\right) \mathrm{d} s\right)$ for notational convenience. Let

$$
M\left(t ; \pi_{1}, \pi_{2}\right)=\Gamma\left(t ; \pi_{1}, \pi_{2}\right) V\left(X^{\pi_{1}, \pi_{2}}(t)\right)+\int_{0}^{t} \Gamma\left(s ; \pi_{1}, \pi_{2}\right) c\left(X^{\pi_{1}, \pi_{2}}(s)\right) \mathrm{d} s .
$$

It is straightforward to check that

$$
M\left(0 ; \pi_{1}, \pi_{2}\right)=V\left(X^{\pi_{1}, \pi_{2}}(0)\right)=V(x), \quad \mathrm{E}_{x}\left[M\left(\tau^{\pi_{1}, \pi_{2}} ; \pi_{1}, \pi_{2}\right)\right]=J^{\pi_{1}, \pi_{2}}(x) .
$$

Itô's formula gives

$$
\begin{aligned}
& M\left(\tau^{\pi_{1}, \pi_{2}} ; \pi_{1}, \pi_{2}\right) \\
& =M\left(0 ; \pi_{1}, \pi_{2}\right)+\int_{0}^{\tau^{\pi_{1}, \pi_{2}}} \Gamma\left(s ; \pi_{1}, \pi_{2}\right)\left[L^{\pi_{1}, \pi_{2}} V\left(X^{\pi_{1}, \pi_{2}}(s)\right)+c-\eta V\left(X^{\pi_{1}, \pi_{2}}(s)\right)\right] \mathrm{d} s \\
& \quad+\int_{0}^{\tau^{\pi_{1}, \pi_{2}}} \Gamma\left(s ; \pi_{1}, \pi_{2}\right)\left[V_{x}\left(X^{\pi_{1}, \pi_{2}}(s)\right) \pi_{1} \sigma_{1} \mathrm{~d} W_{1}(s)-V_{x}\left(X^{\pi_{1}, \pi_{2}}(s)\right) \pi_{2} \sigma_{2} \mathrm{~d} W_{2}(s)\right] .
\end{aligned}
$$

Then

$$
\begin{aligned}
& M\left(\tau^{\pi_{1}, \pi_{2}^{*}} ; \pi_{1}, \pi_{2}^{*}\right) \\
& =M\left(0 ; \pi_{1}, \pi_{2}^{*}\right)+\int_{0}^{\tau^{\pi_{1}, \pi_{2}^{*}}} \Gamma\left(t ; \pi_{1}, \pi_{2}^{*}\right)\left[L^{\pi_{1}, \pi_{2}^{*}} V(X(t))+c-\eta V(X(t))\right] \mathrm{d} t \\
& +\int_{0}^{\tau^{\pi_{1}, \pi_{2}^{*}}} V_{x}\left(X^{\pi_{1}, \pi_{2}^{*}}(t)\right) \pi_{1} \sigma_{1} \mathrm{~d} W_{1}(t)-V_{x}\left(X^{\pi_{1}, \pi_{2}^{*}}(t)\right) \pi_{2}^{*} \sigma_{2} \mathrm{~d} W_{2}(t) \\
& \leq M\left(0 ; \pi_{1}, \pi_{2}^{*}\right)
\end{aligned}
$$

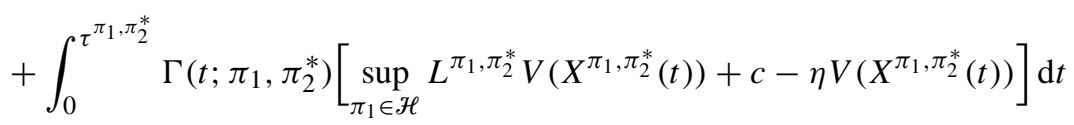

$$
\begin{aligned}
& +\int_{0}^{\tau^{\pi_{1}, \pi_{2}^{*}}} \Gamma\left(t ; \pi_{1}, \pi_{2}^{*}\right)\left[V_{x}\left(X^{\pi_{1}, \pi_{2}^{*}}(t)\right) \pi_{1} \sigma_{1} \mathrm{~d} W_{1}(t)-V_{x}\left(X^{\pi_{1}, \pi_{2}^{*}}(t)\right) \pi_{2}^{*} \sigma_{2} \mathrm{~d} W_{2}(t)\right] \\
& =M\left(0 ; \pi_{1}, \pi_{2}^{*}\right) \\
& +\int_{0}^{\tau^{\pi_{1}, \pi_{2}^{*}}} \Gamma\left(t ; \pi_{1}, \pi_{2}^{*}\right)\left[V_{x}\left(X^{\pi_{1}, \pi_{2}^{*}}(t)\right) \pi_{1} \sigma_{1} \mathrm{~d} W_{1}(t)-V_{x}\left(X^{\pi_{1}, \pi_{2}^{*}}(t)\right) \pi_{2}^{*} \sigma_{2} \mathrm{~d} W_{2}(t)\right] .
\end{aligned}
$$


Since both $\pi_{1}, \pi_{2}^{*} \in[0,1]$ are bounded and $V(x)$ satisfies condition (7), the stochastic integral term in the above inequality is a martingale. Taking the expectation of both sides of the above inequality gives

$$
\mathrm{E}\left[M\left(\tau^{\pi_{1}, \pi_{2}^{*}} ; \pi_{1}, \pi_{2}^{*}\right)\right] \leq M\left(0 ; \pi_{1}, \pi_{2}^{*}\right),
$$

and it is easy to see that the equality holds for $\pi_{1}=\pi_{1}^{*}$, i.e.

$$
\mathrm{E}\left[M\left(\tau^{\pi_{1}^{*}, \pi_{2}^{*}} ; \pi_{1}^{*}, \pi_{2}^{*}\right)\right]=M\left(0 ; \pi_{1}^{*}, \pi_{2}^{*}\right)=V(x) .
$$

Moreover,

$$
\begin{aligned}
J^{\pi_{1}, \pi_{2}^{*}} & =\mathrm{E}\left[M\left(\tau^{\pi_{1}, \pi_{2}^{*}} ; \pi_{1}, \pi_{2}^{*}\right)\right] \\
& \leq M\left(0 ; \pi_{1}, \pi_{2}^{*}\right) \\
& =V(x) \\
& =M\left(0, \pi_{1}^{*}, \pi_{2}^{*}\right) \\
& =\mathrm{E}\left[M\left(\tau^{\pi_{1}^{*}, \pi_{2}^{*}} ; \pi_{1}^{*}, \pi_{2}^{*}\right)\right] \\
& =J^{\pi_{1}^{*}, \pi_{2}^{*}} .
\end{aligned}
$$

Similarly, we can prove that

$$
J^{\pi_{1}^{*}, \pi_{2}^{*}} \leq J^{\pi_{1}^{*}, \pi_{2}} .
$$

Hence, $\left(\pi_{1}^{*}, \pi_{2}^{*}\right)$ is a saddle point strategy for the game by definition (3) and $V(x)$ is identical to the value of the game $J^{\pi_{1}^{*}, \pi_{2}^{*}}(x)$. This completes the proof.

The above theorem guarantees that the solution to (4) and (5) is the value function of the game with the general payoff function. Obviously, the moment condition (7) can be relaxed or replaced by other conditions like that in [2]. Since the basic idea to prove such a verification theorem is similar, we will not consider relaxed conditions in this paper. The following section is an application of Theorem 1 to the stochastic differential game with a particular payoff.

\section{A game of maximizing/minimizing the probability}

In this section we solve the game for a particular payoff. Suppose that player 1 wants to maximize the probability that the difference between two players' surplus reaches the upper barrier before it reaches the lower barrier, while player 2 wants to minimize the same probability. Hence, the game has the payoff function

$$
J^{\pi_{1}, \pi_{2}}(x)=\operatorname{Pr}\left(\tau_{l}^{\pi_{1}, \pi_{2}}>\tau_{u}^{\pi_{1}, \pi_{2}} \mid X^{\pi_{1}, \pi_{2}}(0)=x\right)=\mathrm{E}_{x}\left[\mathbf{1}_{\left\{X^{\pi_{1}, \pi_{2}}\left(\tau^{\pi_{1}, \pi_{2}}\right)=u\right\}}\right] .
$$

Letting $c(x)=\eta(x)=0$ and $g(x)=\mathbf{1}_{\{x=u\}}$ in the Fleming-Bellman-Isaacs equations, (4) and (5) give

$$
\begin{aligned}
& \sup _{\pi_{1} \in[0,1]}\left(D+\pi_{1} \lambda_{1}-\pi_{2}^{*} \lambda_{2}+r x\right) V_{x}(x)+\left(\frac{\sigma_{1}^{2}}{2} \pi_{1}^{2}+\frac{\sigma_{2}^{2}}{2}\left(\pi_{2}^{*}\right)^{2}-\sigma_{1} \sigma_{2} \rho \pi_{1} \pi_{2}^{*}\right) V_{x x}(x)=0, \\
& \inf _{\pi_{2} \in[0,1]}\left(D+\pi_{1}^{*} \lambda_{1}-\pi_{2} \lambda_{2}+r x\right) V_{x}(x)+\left(\frac{\sigma_{1}^{2}}{2}\left(\pi_{1}^{*}\right)^{2}+\frac{\sigma_{2}^{2}}{2} \pi_{2}^{2}-\sigma_{1} \sigma_{2} \rho \pi_{1}^{*} \pi_{2}\right) V_{x x}(x)=0,
\end{aligned}
$$


with the boundary conditions

$$
V(l)=0, \quad V(u)=1 .
$$

If a twice differentiable function $V(x)$ and a pair of numbers $\left(\pi_{1}^{*}, \pi_{2}^{*}\right)$ satisfy (8), (9), (10), and (6), (7), then by the verification theorem, Theorem 1 , we find the value function of the game and the corresponding saddle point strategy.

To obtain a candidate solution to (8) and (9), we consider the following two cases. Since the value function of the game is nondecreasing, we are only interested in functions with the property that $V_{x}(x) \geq 0$ in both cases.

\subsection{Case 1: $V_{x x}<0$}

Given $V_{x x}<0$, the infimum in (9) is reached at $\pi_{2}=0$ or $\pi_{2}=1$. Let

$$
\hat{\pi}_{2}\left(V, \pi_{1}^{*}\right)=\frac{\lambda_{2} V_{x}}{\sigma_{2}^{2} V_{x x}}+\frac{\pi_{1}^{*} \sigma_{1} \rho}{\sigma_{2}} .
$$

Then, for $\hat{\pi}_{2}\left(V, \pi_{1}^{*}\right)>\frac{1}{2}$, the minimizer of the left-hand side of (9) is $\pi_{2}^{*}=0$. Substituting this into (8) gives

$$
\left(D+\pi_{1}^{*} \lambda_{1}+r x\right) V_{x}+\frac{1}{2} \sigma_{1}^{2}\left(\pi_{1}^{*}\right)^{2} V_{x x}=0,
$$

where $\pi_{1}^{*}=\arg \sup _{\pi_{1} \in[0,1]}\left(D+\pi_{1} \lambda_{1}+r x\right) V_{x}+\frac{1}{2} \sigma_{1}^{2} \pi_{1}^{2} V_{x x}$.

Lemma 1. The solution to (12) is

$$
V(x)= \begin{cases}V_{1}:=c_{1}(-D-r x)^{\lambda_{1}^{2} / 2 r \sigma_{1}^{2}+1}+c_{2}, & x \in \Theta_{n, 0,1}, \\ V_{2}:=c_{3} \int_{a}^{x} \exp \left(-\frac{1}{r \sigma_{1}^{2}}\left(D+\lambda_{1}+r y\right)^{2}\right) \mathrm{d} y+c_{4}, & x \in \Theta_{n, 0,2},\end{cases}
$$

where $a$ is a (any) fixed number in $\Theta_{n, 0,2}$, and

$$
\begin{aligned}
& \Theta_{n, 0,1}=\left\{x \in[l, u]:-\frac{\lambda_{1}}{2} \leq D+r x<0,\left(\frac{\lambda_{2}}{\sigma_{2}^{2}}-\frac{\lambda_{1} \rho}{\sigma_{1} \sigma_{2}}\right) \frac{2 \sigma_{1}^{2}}{\lambda_{1}^{2}}(D+r x)>\frac{1}{2}\right\}, \\
& \Theta_{n, 0,2}=\left\{x \in[l, u]: \max \left(-\lambda_{1},\left(\frac{1}{2}-\frac{\sigma_{1} \rho}{\sigma_{2}}\right) \frac{\lambda_{1}^{2} \sigma_{2}^{2}}{2 \lambda_{2} \sigma_{1}^{2}}\right)<D+r x<-\frac{\lambda_{1}}{2}\right\} .
\end{aligned}
$$

The corresponding saddle point strategy, $\left(\pi_{1}^{*}, \pi_{2}^{*}\right)$, is given by

$$
\left(\pi_{1}^{*}, \pi_{2}^{*}\right)= \begin{cases}\left(-\frac{2}{\lambda_{1}}(D+r x), 0\right), & x \in \Theta_{n, 0,1}, \\ (1,0), & x \in \Theta_{n, 0,2},\end{cases}
$$

where the constants $c_{1}, c_{2}, c_{3}$, and $c_{4}$ are determined using the boundary conditions and the smooth conditions.

Proof. Let

$$
\hat{\pi}_{1}=-\frac{\lambda_{1} V_{x}}{\sigma_{1}^{2} V_{x x}} .
$$


Suppose that $\hat{\pi}_{1} \in[0,1]$. Then the maximizer $\pi_{1}^{*}=\hat{\pi}_{1}$. Substituting $\hat{\pi}_{1}$ into (12), it follows that

$$
(D+r x) V_{x}-\frac{\lambda_{1}^{2} V_{x}^{2}}{2 \sigma_{1}^{2} V_{x x}}=0 .
$$

Solving (14) gives the solution $V_{1}$ in (13). Since we assume that $V_{x x}<0$, we conclude that $V_{x}>0$. (If $V_{x}(x)=0$ then $\pi_{1}^{*}(x)=0$ by (19), and, by (18), $\hat{\pi}_{2}(x)=0$, which contradicts the assumption that $\hat{\pi}_{2}>\frac{1}{2}$ given after (18).) Hence, it follows from (14) that $D+r x<0$. Then substituting $V_{1}$ into $\hat{\pi}_{1}$ and letting $\hat{\pi}_{1} \leq 1$ yields $D+r x \geq-\lambda_{1} / 2$. On the other hand, $\hat{\pi}_{2}\left(V_{1}, \hat{\pi}_{1}\right)>\frac{1}{2}$ yields the second inequality in the definition of $\Theta_{n, 0,1}$. Hence, $V_{1}$ is a valid solution when $x \in \Theta_{n, 0,1}$.

If $\hat{\pi}_{1}>1$ then the maximizer of the left-hand side of (12) is 1 . As a result, we have the following equation:

$$
\left(D+\lambda_{1}+r x\right) V_{x}+\frac{1}{2} \sigma_{1}^{2} V_{x x}=0 .
$$

Solving this equation gives the solution $V_{2}$. Again, we conclude that $V_{2}$ is a valid solution when $0<D+\lambda_{1}+r x$ (since $V_{x}>0$ and $V_{x x}<0$ ) and $D+\lambda_{1}+r x<\lambda_{1} / 2$ (by letting $\hat{\pi}_{1}>1$ ). Furthermore, $\hat{\pi}_{2}\left(V_{2}, 1\right)>\frac{1}{2}$ implies that

$$
D+r x>\left(\frac{1}{2}-\frac{\sigma_{1} \rho}{\sigma_{2}}\right) \frac{\lambda_{1}^{2} \sigma_{2}^{2}}{2 \lambda_{2} \sigma_{1}^{2}} .
$$

Hence, $V_{2}(x)$ is a valid solution if $x \in \Theta_{n, 0,2}$. This completes the proof.

For the case in which $\hat{\pi}_{2}\left(V, \pi_{1}^{*}\right) \leq \frac{1}{2}$, the minimizer of the left-hand side of (9) is $\pi_{2}^{*}=1$. As a result of substituting $\pi_{2}^{*}=1$ into (8), $V(x)$ satisfies

$$
\left(D+\pi_{1}^{*} \lambda_{1}-\lambda_{2}+r x\right) V_{x}+\left(\frac{1}{2} \sigma_{1}^{2} \pi_{1}^{*}+\frac{1}{2} \sigma_{2}^{2}-\pi_{1}^{*} \sigma_{1} \sigma_{2} \rho\right) V_{x x}=0,
$$

where

$$
\pi_{1}^{*}=\arg \sup _{\pi_{1} \in[0,1]}\left(D+\pi_{1} \lambda_{1}-\lambda_{2}+r x\right) V_{x}+\left(\frac{1}{2} \sigma_{1}^{2} \pi_{1}^{2}+\frac{1}{2} \sigma_{2}^{2}-\pi_{1} \sigma_{1} \sigma_{2} \rho\right) V_{x x} .
$$

Let $\hat{\pi}_{1}=-\lambda_{1} V_{x} / \sigma_{1}^{2} V_{x x}+\sigma_{2} \rho / \sigma_{1}$, and suppose that $\hat{\pi}_{1} \in[0,1]$. Then

$$
\pi_{1}^{*}=\hat{\pi}_{1}=-\frac{\lambda_{1} V_{x}}{\sigma_{1}^{2} V_{x x}}+\frac{\sigma_{2}}{\sigma_{1}} \rho .
$$

Substituting this into (15) yields

$$
\left(D-\lambda_{2}+r x+\frac{\sigma_{2}}{\sigma_{1}} \lambda_{1} \rho\right) V_{x}+\frac{1}{2} \sigma_{2}^{2}\left(1-\rho^{2}\right) V_{x x}-\frac{1}{2} \frac{\lambda_{1}^{2} V_{x}^{2}}{\sigma_{1}^{2} V_{x x}}=0,
$$

or

$$
\frac{1}{2} \sigma_{2}^{2}\left(1-\rho^{2}\right)\left(\frac{V_{x x}}{V_{x}}\right)^{2}+\left(D-\lambda_{2}+r x+\frac{\sigma_{2}}{\sigma_{2}} \lambda_{1} \rho\right) \frac{V_{x x}}{V_{x}}-\frac{1}{2} \frac{\lambda_{1}^{2}}{\sigma_{1}^{2}}=0 .
$$

It turns out that the solution to (17) is

$$
V_{4}(x):= \begin{cases}c_{7} \int_{d}^{x} \exp \left(\int_{d}^{z} A(s) \mathrm{d} s\right) \mathrm{d} z+c_{8}, & \rho= \pm 1, \\ c_{7}\left(D-\lambda_{2}+r x+\frac{\sigma_{2}}{\sigma_{1}} \lambda_{1} \rho\right)^{\lambda_{1}^{2} / 2 r \sigma_{1}^{2}+1}+c_{8}, & \rho \neq \pm 1,\end{cases}
$$


where

$$
\begin{aligned}
A(x)= & -\frac{\left(D-\lambda_{2}+r x+\sigma_{2} \lambda_{1} \rho / \sigma_{1}\right)}{\sigma_{2}^{2}\left(1-\rho^{2}\right)} \\
& -\frac{\sqrt{\left(D-\lambda_{2}+r x+\sigma_{2} \lambda_{1} \rho / \sigma_{1}\right)^{2}+\sigma_{2}^{2}\left(1-\rho^{2}\right) \lambda_{1}^{2} / \sigma_{1}^{2}}}{\sigma_{2}^{2}\left(1-\rho^{2}\right)},
\end{aligned}
$$

and $d \in[l, u]$ is a fixed point, which will be specified later. The above solution is obtained under the assumption that $\hat{\pi}_{1} \in[0,1]$. In the case $\rho \neq \pm 1$, substituting $V_{4}(x)$ into $0 \leq \hat{\pi}_{1} \leq 1$ yields

$$
\frac{\sigma_{1} \sigma_{2} \rho}{\lambda_{1}} \geq \frac{1}{A(x)} \geq\left(\frac{\sigma_{2}}{\sigma_{1}} \rho-1\right) \frac{\sigma_{1}^{2}}{\lambda_{1}} .
$$

Moreover, $\hat{\pi}_{2}\left(V_{3}, \pi_{1}^{*}\right) \leq \frac{1}{2}$ yields

$$
\left(\frac{\lambda_{2}^{2}}{\sigma_{2}^{2}}-\frac{\lambda_{1} \rho}{\sigma_{1} \sigma_{2}}\right) \frac{1}{A(x)} \leq \frac{1}{2}-\rho^{2} .
$$

Therefore, $V_{4}(x)$ is a valid solution if $x \in \Theta_{n, 1,1}$ and $\rho \neq \pm 1$, where

$$
\Theta_{n, 1,1}=\left\{x \in[l, u]: \frac{\sigma_{1} \sigma_{2} \rho}{\lambda_{1}} \geq \frac{1}{A(x)} \geq\left(\frac{\sigma_{2}}{\sigma_{1}} \rho-1\right) \frac{\sigma_{1}^{2}}{\lambda_{1}},\left(\frac{\lambda_{2}^{2}}{\sigma_{2}^{2}}-\frac{\lambda_{1} \rho}{\sigma_{1} \sigma_{2}}\right) \frac{1}{A(x)}<\frac{1}{2}-\rho^{2}\right\} .
$$

Similarly, for the case in which $\rho= \pm 1$, we can show that $V_{4}(x)$ is a valid solution for $x \in \Theta_{n, 1,1}^{\prime}$, where

$$
\begin{aligned}
\Theta_{n, 1,1}^{\prime}= & \left\{x \in[l, u]: \frac{\lambda_{1} \sigma_{2}}{\sigma_{1}} \min \left(-\rho,-\frac{\rho}{2}\right) \geq D-\lambda_{2}+r x \geq-\frac{\lambda_{1}}{2}\left(1+\frac{\sigma_{2} \rho}{\sigma_{1}}\right),\right. \\
& \left.\left(\frac{\lambda_{2}}{\sigma_{2}^{2}}-\frac{\lambda_{1} \rho}{\sigma_{1} \sigma_{2}}\right) \frac{2 \sigma_{1}^{2}}{\lambda_{1}^{2}}\left(D-\lambda_{2}+r x\right)+\frac{2 \sigma_{1} \lambda_{2} \rho}{\lambda_{1} \sigma_{2}}<\frac{3}{2}\right\} .
\end{aligned}
$$

Then the constant $d$ in (18) is a fixed point in $\Theta_{n, 1,1}$ (if $\rho \neq \pm 1$ ) or $\Theta_{n, 1,1}^{\prime}$ (if $\rho= \pm 1$ ). Finally, substituting $V=V_{4}$ into (16) yields $\pi_{1}^{*}=-\lambda_{1} / \sigma_{1}^{2} A(x)+\sigma_{2} \rho / \sigma_{1}$.

Repeating this procedure, assuming that $\hat{\pi}_{1}>1$ or $\hat{\pi}_{1}<0$ and substituting $\pi_{1}^{*}=1$ or $\pi_{1}^{*}=0$ into (15), respectively, we find solutions for other cases and the corresponding regions where the solutions are valid:

$$
\begin{aligned}
& V_{3}(x):=c_{5} \int_{e_{1}}^{x} \exp \left(-\frac{1}{r \sigma_{2}^{2}}\left(D-\lambda_{2}+r y\right)^{2}\right) \mathrm{d} y+c_{6}, \quad x \in \Theta_{n, 1,0}, \\
& V_{5}(x):=c_{9} \int_{e_{2}}^{x} \exp \left(\frac{-\left(D+\lambda_{1}-\lambda_{2}+r y\right)^{2}}{r\left(\sigma_{1}^{2}+\sigma_{2}^{2}-2 \sigma_{1} \sigma_{2} \rho\right)}\right) \mathrm{d} x+c_{10}, \quad x \in \Theta_{n, 1,2},
\end{aligned}
$$

where

$$
\begin{aligned}
\Theta_{n, 1,0}= & \left\{x: \frac{\lambda_{1} \sigma_{2}}{2 \sigma_{1}} \frac{1}{D-\lambda_{2}+r x}+\rho<0, D-\lambda_{2}+r x>0\right\}, \\
\Theta_{n, 1,2}= & \left\{x: \frac{\lambda_{1}}{2 \sigma_{1}^{2}} \frac{\sigma_{1}^{2}+\sigma_{2}^{2}-2 \sigma_{1} \sigma_{2} \rho}{D+\lambda_{1}-\lambda_{2}+r x}+\frac{\sigma_{2}}{\sigma_{1}} \rho>1, D+\lambda_{1}-\lambda_{2}+r x>0,\right. \\
& \left.-\frac{\lambda_{2}}{2 \sigma_{2}} \frac{\sigma_{1}^{2}+\sigma_{2}^{2}-2 \sigma_{1} \sigma_{2} \rho}{D+\lambda_{1}-\lambda_{2}+r x}<\frac{1}{2}-\frac{\sigma_{1} \rho}{\sigma_{2}}\right\},
\end{aligned}
$$


and the constants $e_{1} \in \Theta_{n, 1,0}$ and $e_{2} \in \Theta_{n, 1,2}$ are fixed points. The above results are summarized in the following lemma.

Lemma 2. The solution to (15) is given by

$$
V(x)= \begin{cases}V_{3}(x), & x \in \Theta_{n, 1,0}, \\ V_{4}(x), & x \in \Theta_{n, 1,1} \text { if } \rho \neq \pm 1, \text { or } x \in \Theta_{n, 1,1}^{\prime} \text { if } \rho= \pm 1, \\ V_{5}(x), & x \in \Theta_{n, 1,2},\end{cases}
$$

and the corresponding saddle point strategy is given by

$$
\left(\pi_{1}^{*}(x), \pi_{2}^{*}(x)\right)= \begin{cases}(0,1), & x \in \Theta_{n, 1,0}, \\ \left(-\frac{\lambda_{1}}{\sigma_{1}^{2} A(x)}+\frac{\sigma_{2}}{\sigma_{1}} \rho, 1\right), & x \in \Theta_{n, 1,1}, \rho \neq \pm 1, \\ \left(-\frac{2}{\lambda_{1}}\left(D-\lambda_{1}+r x\right)-\frac{\sigma_{2}}{\sigma_{1}} \rho, 1\right), & x \in \Theta_{n, 1,1}^{\prime}, \rho= \pm 1, \\ (1,1), & x \in \Theta_{n, 1,2} .\end{cases}
$$

\subsection{Case 2: $V_{x x}>0$}

Under the condition that $V_{x x}>0$, the supremum of the left-hand side of (8) is reached at $\pi_{1}=0$ or $\pi_{1}=1$. Let

$$
\hat{\pi}_{1}\left(V, \pi_{2}^{*}\right)=-\frac{\lambda_{1} V_{x}}{\sigma_{1}^{2} V_{x x}}+\frac{\sigma_{2}}{\sigma_{1}} \rho \pi_{2}^{*}(x) .
$$

If $\hat{\pi}_{1}\left(V, \pi_{2}^{*}\right)>\frac{1}{2}$ then the maximizer of the left-hand side of (8) is $\pi_{1}^{*}=0$, and we obtain the following equation by substituting $\pi_{1}^{*}=0$ into (9):

$$
\left(D-\pi_{2}^{*} \lambda_{2}+r x\right) V_{x}+\frac{1}{2} \sigma_{2}^{2}\left(\pi_{2}^{*}\right)^{2} V_{x x}=0 .
$$

Similarly to (12), (21) has the solution presented in the following lemma.

Lemma 3. The solution to (21) is given by

$$
V(x)= \begin{cases}V_{6}:=c_{11}(D+r x)^{\lambda_{2}^{2} / 2 r \sigma_{2}^{2}+1}+c_{12}, & x \in \Theta_{p, 0,1}, \\ V_{7}:=c_{13} \int_{f}^{x} \exp \left(-\frac{1}{r \sigma_{2}^{2}}\left(D-\lambda_{2}+r y\right)^{2} \mathrm{~d} y\right)+c_{14}, & x \in \Theta_{p, 0,2},\end{cases}
$$

and the corresponding saddle strategy, $\left(\pi_{1}^{*}, \pi_{2}^{*}\right)$, is given by

$$
\left(\pi_{1}^{*}(x), \pi_{2}^{*}(x)\right)= \begin{cases}\left(0, \frac{2}{\lambda_{2}}(D+r x)\right), & x \in \Theta_{p, 0,1}, \\ (0,1), & x \in \Theta_{p, 0,2},\end{cases}
$$

where

$$
\begin{aligned}
& \Theta_{p, 0,1}=\left\{x \in[l, u]: 0<D+r x \leq \frac{\lambda_{2}}{2},\left(-\frac{\lambda_{1}}{\sigma_{1}^{2}}+\frac{\lambda_{2} \rho}{\sigma_{1} \sigma_{2}}\right) \frac{2 \sigma_{2}^{2}}{\lambda_{2}^{2}}(D+r x)>\frac{1}{2}\right\}, \\
& \Theta_{p, 0,2}=\left\{x \in[l, u]: \frac{\lambda_{2}}{2}<D+r x<\lambda_{2},-\frac{\lambda_{1}}{\sigma_{1}^{2}} \frac{2 \sigma_{2}^{2}}{\lambda_{2}^{2}}(D+r x)>\frac{1}{2}-\frac{\sigma_{2}}{\sigma_{1}} \rho\right\},
\end{aligned}
$$

and $f \in \Theta_{p, 0,2}$ is a fixed point. 
If $\hat{\pi}_{1}\left(V, \pi_{2}^{*}\right) \leq \frac{1}{2}$ then the maximizer of the left-hand side of $(8)$ is $\pi_{1}^{*}=1$. As a result of substituting $\pi_{1}^{*}=1$ into (9), we have

$$
\left(D+\lambda_{1}-\pi_{2}^{*} \lambda_{2}+r x\right) V_{x}+\left(\frac{1}{2} \sigma_{1}^{2}+\frac{1}{2} \sigma_{2}^{2}\left(\pi_{2}^{*}\right)^{2}-\sigma_{1} \sigma_{2} \rho \pi_{2}^{*}\right) V_{x x}=0 .
$$

Similarly to (15), (22) has the solution presented in the following lemma.

Lemma 4. The solution to (22) is given by

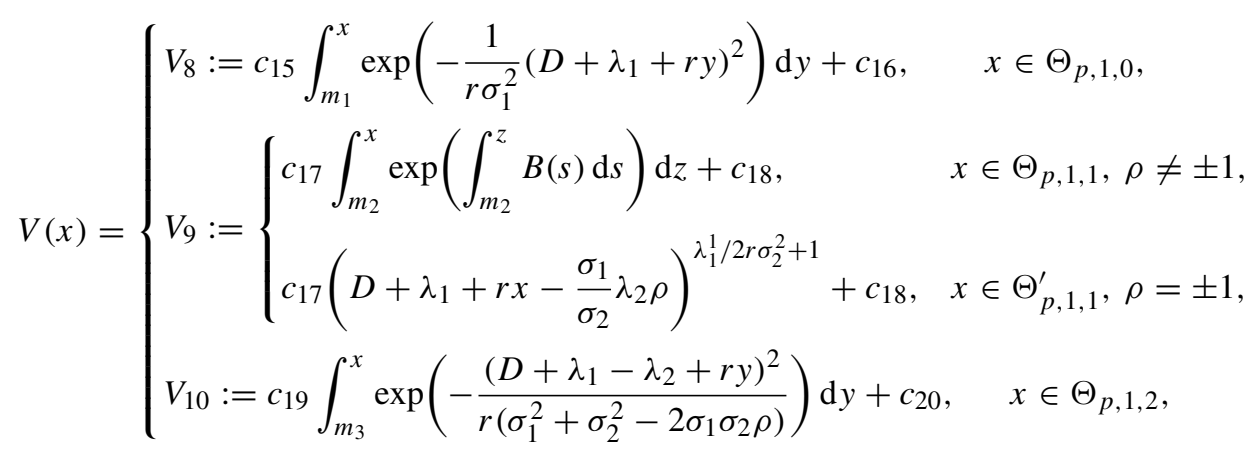

and the corresponding saddle strategy, $\left(\pi_{1}^{*}, \pi^{*}\right)$, is given by

$$
\left(\pi_{1}^{*}(x), \pi_{2}^{*}(x)\right)= \begin{cases}(1,0), & x \in \Theta_{p, 1,0}, \\ \left(1, \frac{\lambda_{2}}{\sigma_{2}^{2}} \frac{1}{B(x)}+\frac{\sigma_{1}}{\sigma_{2}} \rho\right), & x \in \Theta_{p, 1,1}, \rho \neq \pm 1, \\ \left(1, \frac{2}{\lambda_{2}}\left(D+\lambda_{1}+r x\right)-\frac{\sigma_{1}}{\sigma_{2}} \rho\right), & x \in \Theta_{p, 1,1}^{\prime}, \rho= \pm 1, \\ (1,1), & x \in \Theta_{p, 1,2} .\end{cases}
$$

Here

$$
\begin{aligned}
B(s)= & -\frac{\left(D+\lambda_{1}+r x-\sigma_{1} \lambda_{2} \rho / \sigma_{2}\right)}{\sigma_{1}^{2}\left(1-\rho^{2}\right)} \\
+ & \frac{\sqrt{\left(D+\lambda_{1}+r x-\sigma_{1} \lambda_{2} \rho / \sigma_{2}\right)^{2}+\sigma_{1}^{2}\left(1-\rho^{2}\right) \lambda_{2}^{2} / \sigma_{2}^{2}}}{\sigma_{1}^{2}\left(1-\rho^{2}\right)}, \\
\Theta_{p, 1,0}= & \left\{x \in[l, u]:-\frac{\lambda_{2} \sigma_{1}}{2 \sigma_{2}} \frac{1}{D+\lambda_{1}+r x}+\rho<0, D+\lambda_{1}+r x<0\right\}, \\
\Theta_{p, 1,1}= & \left\{x \in[l, u]:-\frac{\sigma_{1} \sigma_{2} \rho}{\lambda_{2}} \leq B(x) \leq\left(1-\frac{\sigma_{1} \rho}{\sigma_{2}}\right) \frac{\sigma_{2}^{2}}{\lambda_{2}},\right. \\
& \left.\left(-\frac{\lambda_{1}}{\sigma_{2}^{2}}+\frac{\lambda_{2} \rho}{\sigma_{1} \sigma_{2}}\right) \frac{1}{B(x)}<\frac{1}{2}-\rho^{2}\right\}, \\
\Theta_{p, 1,1}^{\prime}= & \left\{x \in[l, u]: \frac{\lambda_{2} \sigma_{1}}{\sigma_{2}} \max \left(\rho, \frac{\rho}{2}\right) \leq D+\lambda_{1}+r x \leq \frac{\lambda_{2}}{2}\left(1+\frac{\sigma_{1} \rho}{\sigma_{2}}\right),\right. \\
& \left.\left(-\frac{\lambda_{1}}{\sigma_{1}^{2}}+\frac{\lambda_{2} \rho}{\sigma_{1} \sigma_{2}}\right) \frac{2 \sigma_{2}^{2}}{\lambda_{2}^{2}}\left(D+\lambda \lambda_{1}+r x\right)+\frac{2 \sigma_{1} \lambda_{1}}{\lambda_{2}}>\frac{3}{2}\right\},
\end{aligned}
$$




$$
\begin{aligned}
\Theta_{p, 1,2}= & \left\{x \in[l, u]:-\frac{\lambda_{2}}{\sigma_{2}^{2}} \frac{0.5 \sigma_{1}^{2}+0.5 \sigma_{2}^{2}-\sigma_{1} \sigma_{2} \rho}{D+\lambda_{1}-\lambda_{2}+r x}+\frac{\sigma_{1}}{\sigma_{2}} \rho>1,\right. \\
& \left.D+\lambda_{1}-\lambda_{2}+r x<0, \frac{\lambda_{1}}{2 \sigma_{1}^{2}} \frac{\sigma_{1}^{2}+\sigma_{2}^{2}-2 \sigma_{1} \sigma_{2} \rho}{D+\lambda_{1}-\lambda_{2}+r x}<\frac{1}{2}-\frac{\sigma_{2}}{\sigma_{1}} \rho\right\},
\end{aligned}
$$

and $m_{1}, m_{2}$, and $m_{3}$ are fixed constants that belong to the corresponding sets.

With some tedious calculations we can show that $\Theta_{n, 0,1}, \Theta_{n, 0,2}, \Theta_{n, 1,0}, \Theta_{n, 1,1}$ (or $\Theta_{n, 1,1}^{\prime}$ if $\rho= \pm 1$ ), $\Theta_{n, 1,2}$ and $\Theta_{p, 0,1}, \Theta_{p, 0,2}, \Theta_{p, 1,0}, \Theta_{p, 1,1}\left(\right.$ or $\Theta_{p, 1,1}^{\prime}$ if $\rho= \pm 1$ ), $\Theta_{p, 1,2}$ are mutually exclusive and their union is $[l, u]$. Depending on the model parameters $\left(\mu_{i}, \lambda_{i}, \sigma_{i}, i=1,2\right)$ and the correlation coefficient $\rho$ between the two surplus processes, some sets may be empty.

It is straightforward to verify that the above lemmas provide a solution to the FlemingBellman-Isaacs equations (8) and (9) for all $x \in[l, u]$. The function $V_{x}(x)$ is smooth on all sets, so it is bounded on $[l, u]$. Therefore, condition (7) is satisfied and $V(x)$ obtained above is the value of the game achieved at the saddle point $\left(\pi_{1}^{*}, \pi_{2}^{*}\right)$ by Theorem 1 . In the following section we deal with the particular case $\rho \leq 0$ and show the structure of the solution clearly.

\section{A numerical example: the $\rho \leq 0$ case}

Provided that the condition $\rho<0$ holds, some sets defined previously are simplified or degenerated:

$$
\begin{aligned}
& \Theta_{n, 0,1}=\varnothing, \\
& \Theta_{n, 0,2}=\varnothing, \\
& \Theta_{n, 1,0}=\left\{x_{2}<x\right\}, \\
& \Theta_{n, 1,1}=\left\{\frac{\sigma_{1} \sigma_{2} \rho}{\lambda_{1}} \geq \frac{1}{A(x)} \geq\left(\frac{\sigma_{2} \rho}{\sigma_{1}}-1\right) \frac{\sigma_{1}^{2}}{\lambda_{1}}\right\}=\left\{x_{1} \leq x \leq x_{2}\right\}, \\
& \Theta_{n, 1,2}=\left\{0<D+\lambda_{1}-\lambda_{2}+r x<\frac{\lambda_{1}\left(\sigma_{1}^{2}+\sigma_{2}^{2}-2 \sigma_{1} \sigma_{2} \rho\right)}{2 \sigma_{1}^{2}\left(1-\sigma_{2} \rho / \sigma_{1}\right)}\right\}=\left\{x_{0}<x<x_{1}\right\},
\end{aligned}
$$

where

$$
\begin{aligned}
& x_{0}=\frac{1}{r}\left(\mu_{2}-\mu_{1}\right), \\
& x_{1}=\frac{1}{r}\left(\mu_{2}-\mu_{1}+\frac{\lambda_{1}}{2 \sigma_{1}^{2}\left(1-\sigma_{2} \rho / \sigma_{1}\right.}\left(\sigma_{1}^{2}+\sigma_{2}^{2}-2 \sigma_{1} \sigma_{2} \rho\right)\right), \\
& x_{2}=\frac{1}{r}\left(\lambda_{2}-D-\frac{\lambda_{1} \sigma_{2}}{2 \sigma_{1} \rho}\right) .
\end{aligned}
$$

Similarly, we obtain

$$
\begin{aligned}
& \Theta_{p, 0,1}=\varnothing, \\
& \Theta_{p, 0,2}=\varnothing, \\
& \Theta_{p, 1,0}=\left\{x<x_{2}^{\prime}\right\}, \\
& \Theta_{p, 1,1}=\left\{x_{2}^{\prime} \leq x \leq x_{1}^{\prime}\right\}, \\
& \Theta_{p, 1,2}=\left\{x_{1}^{\prime}<x<x_{0}\right\},
\end{aligned}
$$


where

$$
\begin{aligned}
& x_{1}^{\prime}=\frac{1}{r}\left(\mu_{2}-\mu_{1}-\frac{\lambda_{2}}{2 \sigma_{2}^{2}\left(1-\sigma_{1} \rho / \sigma_{2}\right)}\left(\sigma_{1}^{2}+\sigma_{2}^{2}-2 \sigma_{1} \sigma_{2} \rho\right)\right), \\
& x_{2}^{\prime}=\frac{1}{r}\left(-\lambda_{1}-D+\frac{\lambda_{2} \sigma_{1}}{2 \sigma_{2} \rho}\right) .
\end{aligned}
$$

Note that $V_{5}(x)$ on $\Theta_{n, 1,2}$ and $V_{10}(x)$ on $\Theta_{p, 1,2}$ have the same form, disregarding the constants, and $\Theta_{n, 1,2} \cup \Theta_{p, 1,2}=\left\{x_{1}^{\prime}<x<x_{0}\right\} \cup\left\{x_{0}<x<x_{1}\right\}$. Since the value function is continuous and smooth, we conclude that the function has the same form at $x_{0}$ (actually, $x_{0}$ is an inflection point) and we may keep only one of $V_{5}$ and $V_{10}$ on $\left\{x_{1}^{\prime}<x<x_{1}\right\}$ (this simplification is actually generally correct for all $\rho \in[-1,1]$ ). Then applying Lemmas $2-5$, the solution to the Fleming-Bellman-Isaacs equations (8) and (9) is given by

$$
V(x)= \begin{cases}V_{3}=c_{5} \int_{x_{2}}^{x} \exp \left(-\frac{1}{r \sigma_{2}^{2}}\left(D-\lambda_{2}+r y\right)^{2}\right) \mathrm{d} y+c_{6}, & x>x_{2}, \\ V_{4}=c_{7} \int_{x_{1}}^{x} \exp \left(\int_{x_{1}}^{z} A(s) \mathrm{d} s\right) \mathrm{d} z+c_{8}, & x_{1} \leq x \leq x_{2}, \\ V_{5}=c_{9} \int_{x_{0}}^{x} \exp \left(\frac{-\left(D+\lambda_{1}-\lambda_{2}+r y\right)^{2}}{r\left(\sigma_{1}^{2}+\sigma_{2}^{2}-2 \sigma_{1} \sigma_{2} \rho\right)}\right) \mathrm{d} y+c_{10}, & x_{1}^{\prime}<x<x_{1}, \\ V_{9}=c_{17} \int_{x_{2}^{\prime}}^{x} \exp \left(\int_{x_{2}^{\prime}}^{z} B(s) \mathrm{d} s\right) \mathrm{d} z+c_{18}, & x_{2}^{\prime} \leq x \leq x_{1}^{\prime}, \\ V_{8}=c_{15} \int_{x_{2}^{\prime}}^{x} \exp \left(-\frac{1}{r \sigma_{1}^{2}}\left(D+\lambda_{1}+r y\right)^{2}\right) \mathrm{d} y+c_{16}, & x<x_{2}^{\prime},\end{cases}
$$

and the corresponding saddle point strategy is given by

$$
\left(\pi_{1}^{*}, \pi_{2}^{*}\right)= \begin{cases}(0,1), & x>x_{2}, \\ \left(-\frac{\lambda_{1}}{\sigma_{1}^{2} A(x)}+\frac{\sigma_{2}}{\sigma_{1}} \rho, 1\right), & x_{1} \leq x \leq x_{2}, \\ (1,1), & x_{1}^{\prime}<x<x_{1}, \\ \left(1, \frac{\lambda_{2}}{\sigma_{2}^{2} B(x)}+\frac{\sigma_{1}}{\sigma_{2}} \rho\right), & x_{2}^{\prime} \leq x \leq x_{1}^{\prime}, \\ (1,0), & x<x_{2}^{\prime},\end{cases}
$$

where the constants $c_{5}, \ldots, c_{10}, c_{15}, \ldots, c_{18}$ are determined by the boundary conditions $V(l)=$ 0 and $V(u)=1$ and the smooth conditions

$V_{3}\left(x_{2}\right)=V_{4}\left(x_{2}\right), \quad V_{3}^{\prime}\left(x_{2}\right)=V_{4}^{\prime}\left(x_{2}\right), \quad \ldots, \quad V_{9}\left(x_{2}^{\prime}\right)=V_{10}\left(x_{2}^{\prime}\right), \quad V_{9}^{\prime}\left(x_{2}^{\prime}\right)=V_{10}^{\prime}\left(x_{2}^{\prime}\right)$.

In Figures 1 and 2 we present numerical examples of the value function and the saddle point strategy, respectively. We select parameters artificially so that each function is significantly visible on each interval. For example, we choose relatively large $\sigma_{1}$ and $\sigma_{2}$ in order to make $V_{3}$ and $V_{8}$ significant, and we choose $l$ and $u$ in order to show as many intervals as possible. Figure 1 shows that $V_{8}(x)$ and $V_{9}(x)$ are convex, $V_{5}(x)$ is convex when $x<x_{0}$ and concave when $x>x_{0}$, and $V_{4}(x)$ and $V_{3}(x)$ are concave. All of these functions are connected smoothly. Figure 2 shows that player 1 tends to take the highest risk exposure $\left(\pi_{1}^{*}=1\right.$, i.e. no reinsurance) when $x$, the difference between two surplus processes, is at a relatively low level. The player 


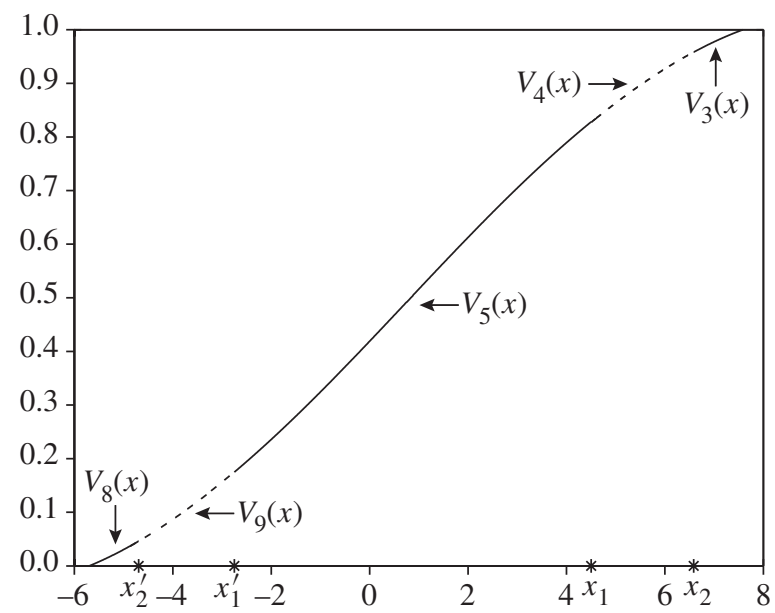

FIgURE 1: The value function of the maximizing probability game. The parameters used are $\mu_{1}=0.1$, $\mu_{2}=0.13, \lambda_{1}=0.15, \lambda_{2}=0.14, \sigma_{1}=\sigma_{2}=0.75, r=0.04, \varphi=-0.9, l=-5.6944, u=7.5833$, $x_{2}^{\prime}=-4.6944, x_{1}^{\prime}=-2.75, x_{1}=4.5$, and $x_{2}=6.5833$.
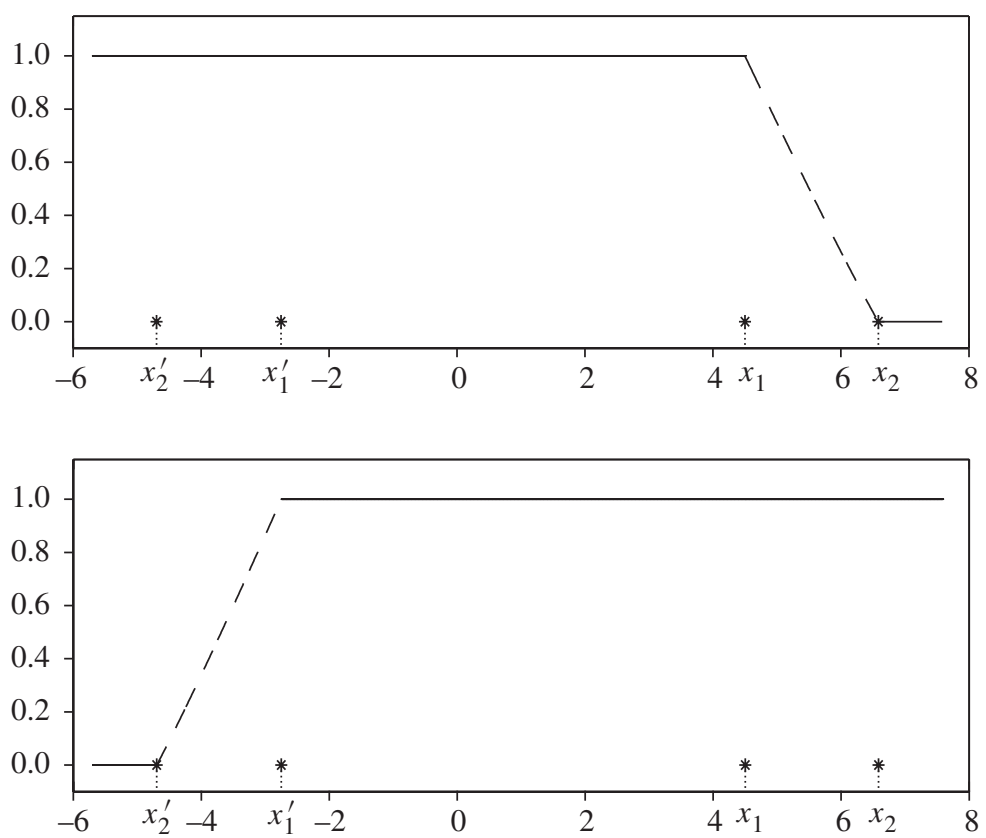

FIGURE 2: The saddle point strategies of player 1 (top) and player 2 (bottom).

will take the lowest risk exposure ( $\pi_{1}^{*}=0$, i.e. full reinsurance) when $x$ is at a relatively high level. If $x$ is between $x_{1}$ and $x_{2}$, the saddle point strategy of player 1 is no longer simple. Although its curve looks like a straight line in the figure, the strategy is a complicated function of $x$ and equals $-\lambda_{1} / \sigma_{1}^{2} A(x)+\sigma_{2} \rho / \sigma_{1}$. For player 2, the strategy is an almost mirror image of that for player 1 . 
For the case in which $\rho=0$, the solution is obtained by letting $\rho \rightarrow 0^{-}$in (23). Direct calculations show that $x_{2}=\infty$ and $x_{2}^{\prime}=-\infty$, and, hence, the solution is constructed by $V_{4}$, $V_{5}$, and $V_{9}$ only.

\section{Conclusions and summary}

In this paper we studied a zero-sum stochastic differential game between two insurance companies who compete on their surplus processes when they make decisions on reinsurance. One company tries to maximize a payoff function depending on the difference between their surplus processes, while the other company tries to simultaneously minimize the same quantity. We studied the problem for a general payoff function and provided a verification theorem for the solution to the Fleming-Bellman-Isaacs equations. Furthermore, we studied the maximizing/minimizing probability game as an application of the theorem. We showed that, for such a game, at least one player's saddle point strategy takes extreme or trivial values, that is, the reinsurance proportion is either 1 or 0 , while it takes nontrivial values on the interval $\left[x_{1}, x_{2}\right]$ or $\left[x_{2}^{\prime}, x_{1}^{\prime}\right]$, during the whole competition.

Although we assumed that the insurance companies invest only in a risk-free asset, it is straightforward to extend the result in this paper to the case where a risky asset is involved. However, if two risky assets with different volatilities are added into the model, it will be difficult to obtain explicit solutions in general. The verification theorem might still hold though. In this paper we considered competition between two noncooperative insurance companies. As a direct extension, a topic of further study could be cooperative games among insurers, between an insurer and a reinsurer, or an individual person and an insurer.

\section{Acknowledgements}

The author is grateful to Professor M. Taksar for many helpful discussions on reinsurance models. The author would also like to thank the anonymous referee for a careful reading and excellent suggestions.

\section{References}

[1] Basar, T. and Olsder G. J. (1982). Dynamic Noncooperative Game Theory. Academic Press, London.

[2] Browne, S. (2000). Stochastic differential portfolio games. J. Appl. Prob. 37, 126-147.

[3] Elliott, R. J. (1976). The existence of value in stochastic differential games. SIAM J. Control Optimization 14, 85-94.

[4] Elliott, R. J. And Davis, M. H. A. (1981). Optimal play in a stochatic differential game. SIAM J. Control Optimzation 19, 543-554.

[5] Fleming, W. H. and Rishel, R. W. (1975). Deterministic and Stochastic Optimal Control. Springer, Berlin.

[6] Klugman, S. A., Panjer, H. H. and Willmot, G. E. (2004). Loss Models, 2nd edn. John Wiley, Hoboken, NJ.

[7] Krylov, N. V. (1980). Controlled Diffusion Processes. Springer, New York.

[8] Suijs J., De Waegenaere, A. And Borm, P. (1998). Stochastic cooperative games in insurance. Insurance Math. Econom. 22, 209-228.

[9] TAKsaR, M. I. (2000). Optimal risk and dividend distribution control models for an insurance company. Math. Meth. Operat. Res. 51, 1-42.

[10] TAKSAR, M. I. AND MarkusSEN, C. (2003). Optimal dynamic reinsurance policies for large insurance portfolios. Finance Stoch. 7, 97-121.

[11] Yeung, D. W. K. and Petrosyan, L. A. (2006). Cooperative Stochastic Differential Games. Springer, New York.

[12] ZENG, X. (2010). Optimal reinsurance with a rescuing procedure. Insurance Math. Econom. 46, 397-405. 